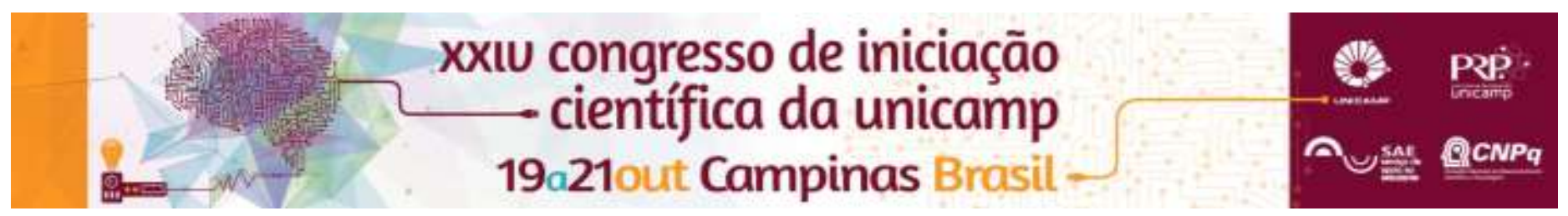

\title{
Micro-CT study of anatomy of humans permanents canines
}

\section{Fernanda M. Antonialli, Alexandre R. Freire, Felippe B. Prado, Ana Cláudia Rossi.}

\begin{abstract}
In this study, we evaluated the external and internal anatomy of permanent human canines by computed microtomography analysis. Here, we hope to find possible variations in the morphology of the teeth analyzed.
\end{abstract}

\section{Key words: \\ morphology, dental anatomy, microtomography.}

\section{Introduction}

The ausence of knowledge of the external and internal morphology of teeth is a major cause of failure in endodontic therapy. It is possible to find canals varying in number, size, diameter, set of teeth with different fusions, directions and stages of development. Thus, the total knowledge of the internal and external morphology of the teeth becomes mandatory in clinical and radiographic evaluation by dentist ${ }^{1}$.

The lower canine present a internal anatomy that usually accompanies the external anatomy; so the pulp chambers follow the shape of their crowns, but the root canals usually have a flatness in the mesial-distal direction and thus widening the buccolingual direction, especially in the middle third. Here the root canal may bifurcate, not following the external anatomy of the root, a small island of dentin is formed between the buccal and lingual channels; in most cases channels are merged again and terminating in a single apical foramen. But there is a variability in the final thirds of the root ${ }^{2}$.

Currently, computed microtomography (micro-CT) is being used to evaluate the dental anatomy due to its high resolution and no destruction of the specimens.

The aim of this study was evaluated the external and internal anatomy of permanent human lower canines by computed microtomography analysis.

\section{Results and Discussion}

30 permanent lower canine were scanned in a SkyScan 1174 microtomography (SkyScan, Leuven, Belgium). After scanning, the images were exported to NRecon reconstruction software (SkyScan, Leuven, Belgium), which was reconstructed three-dimensionally. The images were transferred to the Mimics software v. 17 (Materialise, Belgium), for analysis of the internal and external anatomy of the roots of the lower canines. The internal anatomy of the roots were evaluated according to the root canal system and shape the root canal (cervical, medium and apical third) ${ }^{3}$. The external anatomy of the teeth was evaluated according radicular longitudinal sulcus. A descreptive statistical analysis was performed.

In results, we obtained that the most frequent (86.66\%), was the Type I (single canal) followed by the Type III (a canal that splits into two within the root, joining in a canal in apical) with $13.3 \%$.

Regarding the shape of the cervical third canal was more frequent circular shape $(56.66 \%)$, followed by oval $(40 \%)$ and finally the halter shape (3.33\%).

In the middle third of the oval and halter shape showed the frequency in $13.33 \%$ and $43.33 \%$, respectvely. In apical the most common shape was circular $(96.66 \%)$, followed by oval shape (3.33). In external anatomy, the presence radicular longitudinal sulcus was the $86.66 \%$.

\section{Conclusions}

In conclusion, the permanent lower canine has anatomical variations, which apply the canal type. The most common canal is type I and in the cervical third, is more circular, alternating between oval and halter shape in the middle third and presenting circular shape in the apical third.

These finds contribute to a better understanding of the internal and external anatomy of the lower canine, that is important for the clinical areas as endodontics treatments.

\section{Acknowledgement}

We are grateful to "National Counsel of Technological and Scientific Development" and University of Campinas for financial support.

\footnotetext{
${ }^{1}$ Martos, J.; Lubian, C.; Silveira, L.F.M.; Castro, L.A.S.; Luque, C.M.F., J. Endod. 2010, 36, 664-667.

${ }^{2}$ Kaffe, I.; Kaufman, A.; Littner, M.M.; Lazarson, A., Int. Endod. J. 1985, 18, 253-9.

3 Vertucci, F.J., J. Am. Dent. Assoc., 1974, 89, 369-71.
} 